\title{
Afterword: A Call to Action
}

Ronica Mukerjee, Randi Singer, Linda Wesp, and Pia Pauline Lenon

As we write this conclusion, it is July of 2020 and our world as we knew it is both exactly the same and entirely different from when we began writing this book several years ago. The systems we work within continue to be shaped by intersecting systems of oppression. Voices rising against these systems are louder, but dismantling of the systems still needs to happen on every level.

Our biggest disappointment, now more than ever, would be that clinicians will read this text, close the book, and continue their usual method of care without initiating new actions to increase social justice for their patients. We are worried that you, our colleagues, will ask for more learning opportunities, more reading groups, and more continuing education, but that you won't do the necessary personal work to transform yourselves. That work, in conjunction with outward positive action, is exactly what is required, and although it should have started a long time ago, starting immediately will do. With privilege that comes from being in healthcare leadership roles comes the ability to forget and/or to simplify solutions. It is easier for us as faculty, staff, and students to say, "We understand the problems," but then shrug our shoulders and say that the system is too difficult to change. We implore you to remain uncomfortable.

Employ your knowledge of the five tenets of cultural safety: partnerships, personal activities of daily living (ADLs), prevention of harm, patient centering, and purposeful self-reflection (see Chapter 1). Utilizing this framework, providers will shift the power balance to patients through learning, through engaging in the struggle for body and community autonomy, through challenging police aggression and other biased violence, and through putting our brains and intellects and selves on the line too as much as is needed to create real, conscientious, and relevant change.

If this book has made a lasting impression on you, you will find yourself asking more complex questions than you will have simple answers. How do we turn an oppressive system into an opportunity for change? How do we restructure culturally and medically negligent healthcare systems into ones that reflect appropriate care for the most excluded people in healthcare? Patients who identify as part of the LGBTQIA+ population, particularly BIPOC (black, indigenous, people of color) people, experience a multitude of barriers to obtaining healthcare, and many of these barriers stem from providers' lack of knowledge regarding the type of care patients require and how to approach patients. But these barriers are also inherently a part of our larger healthcare system-a system that has been designed within a framework of 
historical oppression. The system is not broken; it is working exactly as it has been designed, having been informed by heteropatriarchy, white supremacy, and capitalism. We must continue to ask complex questions about how we can completely dismantle and reimagine a system of care; that dismantling and recreation from the ashes is the goal, not the pipe dream. We must call out the exclusion of justice that is too often perpetuated on black and brown people, even when it does not advance our careers. The system as designed will continue to propagate a matrix of oppression. We ask you to consider instead that our healthcare system is doing what it has been designed to do-and therefore it must be completely dismantled, reimagined, and redesigned through a lens of racial, economic, and health justice.

At the same time, we must commit to changing the experiences that patients have with you, one on one, in the clinical setting. We as clinicians must take purposeful steps right now to build safe and trusting relationships that include activism and raising our voices to those in positions of power, in ways that should take you out of your comfort zone. This pushing against a comfort zone means that we (clinicians) are not "just here to help." We are instead here to ensure that LGBTQIA+ and BIPOC patients feel safe and entrust us with their care, knowing that we are working to create change. With such a mindset, every story they want validated, acknowledged, and seen in our clinical visits can be escalated to inform how we reimagine and redesign healthcare so the system can become inherently safe. We must not only work directly with prison healthcare providers and corrections officers to change the status quo, but we must abolish prisons. We must not only commit to spending hours on the phone with insurance companies, surgeons, and specialists to ensure essential care for those most marginalized, stigmatized, and forgotten by a system of oppression, but we must have universal healthcare.

We need to create change by:

1. Understanding the history of the pathologization that LGBTQIA+ people, particularly BIPOC people, have undergone, and the current stigma these populations experience, so that we can work to strategically address barriers

2. Creating further inclusion of LGBTQIA+ populations through provider alignment with culturally safe as well as evidence-based practices in these populations

3. Consistently accepting corrections from patients when it comes to language regarding definitions and naming of their genders, sexualities, identities, and bodies

4. Ensuring that the patient's health concerns for coming into the healthcare setting are addressed as the patient states and not the ways that create greater provider comfort

5. Utilizing this book to understand a trauma-informed approach when taking healthcare interviews and conducting physical exams in order to avoid retraumatization and allow for the greatest environment of safety by the patient

6. Recognizing the individualized and specialized needs of transgender and gender expansive (TGE) individuals during the perinatal period, including emotional and cultural needs 
7. Listening and appropriately responding to your patients and their experiences

8. Choosing racial and economic justice and body autonomy as crucial points of activism, foment, and advocacy, particularly with affected communities

Despite the advice and resources this book has offered, it is important to keep in mind that this book solely offers a framework and guide. It is not a protocol for the care of patients. Much like in the context of care of different ethnic backgrounds or of any patients, for that matter, the care of LGBTQIA+ including BIPOC patients still differs from patient to patient. Understand that each patient has their own distinct narrative; a narrative that the provider must explore with the patient in order to understand how to best care for them. Receiving good healthcare is a human necessity, and if we actually believe that, we must put our intellects, words, compassions, deeds, intentions, careers, and selves on the line to ensure its best delivery. 